\title{
Superação de Dormência de Sementes de Albizia pedicellaris (DC.) L. Rico
}

\author{
Juliana Müller Freire ${ }^{1}$, Danilo Henrique dos Santos Ataíde², \\ Janaína Ribeiro Costa Rouws ${ }^{1}$ \\ ${ }^{1}$ Embrapa Agrobiologia, Seropédica/RJ, Brasil \\ ${ }^{2}$ Instituto de Florestas, Universidade Federal Rural do Rio de Janeiro - UFRRJ, Seropédica/RJ, Brasil
}

\begin{abstract}
RESUMO
O trabalho teve como objetivo avaliar a eficiência de métodos de quebra de dormência em sementes de Albizia pedicellaris em laboratório. Foram aplicados 10 tratamentos pré-germinativos: escarificação química com imersão em ácido sulfúrico por 5, 10, 15 e 30 minutos, escarificação física, seguida ou não de embebição em água por 6, 12 e 24 horas, choque térmico e testemunha. Foi utilizado o delineamento inteiramente casualizado com 4 repetições, com 25 sementes por unidade experimental. Os seguintes parâmetros foram avaliados: percentual de germinação, índice de velocidade de germinação, número de plântulas normais, número de plântulas anormais, mortalidade. Com base nos resultados indicam-se como tratamentos para quebra de dormência da A. pedicellaris a escarificação mecânica seguida ou não de embebição por 6 ou 12 horas ou a imersão em ácido sulfúrico por 30 minutos.
\end{abstract}

Palavras-chave: germinação, sementes florestais nativas, sementes duras.

\section{Dormancy breaking of Albizia pedicellaris (DC.) L. Rico seeds}

\begin{abstract}
The study aimed to evaluate the efficiency of seeds dormancy breakage methods for Albizia pedicellaris in laboratory. Seeds were treated with ten pre-sowing treatments: chemical scarification using sulfuric acid for 5, 10, 15 and 30 minutes, physical scarification followed or not by immersion in water for 6, 12 and 24 hours, thermal shock and control. Completely randomized design was used with four replications of 25 seed per experimental unit. The followings variables were evaluated: germination percentage, speed germination index, normal seedlings number, abnormal seedling number, and mortality. The results indicate that the most efficient method to overcome dormancy was mechanical scarification followed or not by immersion for 6 or 12 hours or immersion in sulfuric acid for 30 minutes.
\end{abstract}

Keywords: germination, native forest seed, dormant seed. 


\section{INTRODUÇÃO}

Usualmente conhecida como balízia, cambuí preto ou juerana branca, Albizia pedicellaris (DC.) L. Rico é uma espécie arbórea semidecídua nativa do Brasil pertencente à família Fabaceae que apresenta crescimento rápido, sendo classificada como pioneira (Lorenzi, 2002). Tem distribuição natural no Brasil, Equador, Guiana Francesa, Suriname, Venezuela (Funk et al., 2007; Jorgensen \& León-Yánez, 1999; Hokche et al., 2008; Steyermark, 1995). No Brasil ocorre na mata pluvial de terra firme na Região Amazônica, Cerrado e Mata Atlântica, com ampla distribuição geográfica (Iganci, 2014). Suas raízes apresentam capacidade de associação com bactérias diazotróficas (Faria, 1995) e consequente aporte de nitrogênio no solo, sendo uma espécie potencial para utilização em programas de recuperação de áreas degradadas por mineração (Franco et al., 1995), tolerando tanto o plantio em áreas sujeitas a alagamento quanto em terra firme.

As sementes de Albizia pedicellaris, assim como de outras espécies arbóreas da família Fabaceae apresentam dormência física, causada pela impermeabilidade do tegumento (Villiers, 1972; Rolston, 1978; Souza et al., 2015). A impermeabilidade à água pode ser causada pela presença de substâncias químicas como suberina, lignina, cutina, taninos, que podem estar presentes em diferentes partes da semente, como na testa, pericarpo e membrana nuclear, características que diferem de uma espécie para outra (Mayer \& Poljakoff-Mayber, 1982). Essa característica requer a aplicação de tratamentos específicos para promoção da germinação em curto espaço de tempo, pois se, por um lado, a dormência é vantajosa para a sobrevivência das espécies em condições naturais, por outro ela é prejudicial à atividade de produção de mudas em viveiros, na qual se deseja que grandes quantidades de sementes germinem em curto espaço de tempo, permitindo a produção de mudas uniformes (Mello, 1998).

A escarificação química, realizada com ácido sulfúrico, assim como a escarificação mecânica (abrasão das sementes sobre uma superfície áspera) e tratamentos térmicos, como imersão em água a diferentes temperaturas, são comumente utilizados para superação desse tipo de dormência, pois proporcionam o desgaste do tegumento e aumentam a sua permeabilidade, permitindo a troca de água e gases (Floriano, 2004; Souza et al., 2007).
A aplicação de tratamentos pré-germinativos, quando feita de forma eficiente, não danifica o embrião $e$ viabiliza a germinação. Esse mesmo procedimento é promovido na natureza por fendas abertas na casca por ação de microorganismos, fungos ou ácidos fracos do solo. Entretanto, para cada espécie é necessária a realização de estudos quanto ao tipo de tratamento e tempo de imersão da semente no ácido ou água, devido à variação na composição e na espessura dos tegumentos (Santos et al., 2011; Giachini et al., 2010; Costa et al., 2010; Cruz et al., 2007). Essa variação também pode ocorrer entre lotes e procedências de uma mesma espécie (Berger et al., 2014; Ladeia et al., 2012; Martins \& Nakagawa, 2008; Alves et al., 2005a; Botezelli et al., 2000).

Estudos sobre quebra de dormência de sementes com a utilização de métodos de escarificação mecânica, química e térmica já foram realizados para várias espécies do gênero Albizia sp, como Albizia richardiana King and Prain (Azad et al., 2010), Albizia procera (Roxb.) Benth (Azad et al., 2012; Sajeevukumar et al., 1995), Albizia hasslerii (Chod.) Burkart (Kissmann et al., 2009), Albizia falcataria (L.) Fosberg (Sajeevukumar et al., 1995), Albizia schimperiana Oliv. (Msanga \& Maghembe, 1986). Entretanto, nenhum estudo foi publicado ainda para Albizia pedicellaris, razão do presente trabalho ter como objetivo estudar diferentes métodos para quebra de dormência de sementes desta espécie.

\section{MATERIAL E MÉTODOS}

As sementes utilizadas no experimento foram colhidas em julho de 2010 em Porto de Trombetas, PA, município de Oriximiná, coordenadas $1^{\circ} 40^{\prime} 30^{\prime \prime}$ $56^{\circ} 27^{\prime} 13^{\prime \prime}$ e foram transportados para a Embrapa Agrobiologia (Seropédica, RJ), onde permaneceram um ano estocadas em câmara fria a $15^{\circ} \mathrm{C}$. Após esse período, foi determinado o teor de umidade do lote pelo método de estufa, $105 \pm 3^{\circ} \mathrm{C}$ por 24 horas, adotando-se duas repetições contendo $5 \mathrm{~g}$ de semente cada, e também o peso de mil sementes, que consistiu na pesagem de oito amostras de 100 sementes (Brasil, 2009).

Para implantação do teste de germinação, primeiramente realizou-se a assepsia das sementes através de imersão em hipoclorito de sódio $5 \%$ $(\mathrm{v} / \mathrm{v})$ por 10 minutos, seguida de lavagem em água 
corrente. Os tratamentos utilizados para a superação da dormência foram:

Testemunha $\left(\mathrm{T}_{1}\right)$ - Sem nenhum tratamento, sementes intactas.

Choque térmico $\left(\mathrm{T}_{2}\right)$ - Foi feita a imersão das sementes em água na temperatura de $80{ }^{\circ} \mathrm{C}$ por 1 minuto, sendo em seguida lavadas em água corrente na temperatura ambiente.

Escarificação mecânica - Friccionou-se manualmente a região oposta ao eixo do embrião sobre lixa de massa n. 150, até se obter uma leve mudança de cor nessa área do tegumento; logo após, as sementes foram colocadas para embeber por $6 \mathrm{~h}\left(\mathrm{~T}_{3}\right), 12 \mathrm{~h}\left(\mathrm{~T}_{4}\right)$ e 24 $\mathrm{h}\left(\mathrm{T}_{5}\right)$ e sem embebição $\left(\mathrm{T}_{6}\right)$.

Escarificação química - As sementes foram imersas em ácido sulfúrico na concentração $98 \%$ por: $5 \mathrm{~min}$ $\left(\mathrm{T}_{7}\right), 10 \min \left(\mathrm{T}_{8}\right), 15 \min \left(\mathrm{T}_{9}\right)$ e $30 \min \left(\mathrm{T}_{10}\right)$, sendo posteriormente lavadas em água corrente.

Após tratadas, realizou-se a semeadura sobre substrato de areia e vermiculita (1:1), ambos previamente autoclavados, em recipientes gerbox transparentes com tampa $(11 \times 11 \times 3,5 \mathrm{~cm})$. O delineamento experimental utilizado foi o inteiramente casualizado, com 4 repetições e 25 sementes por unidade experimental. Efetuou-se em seguida a irrigação do substrato, que consistiu em adicionar um volume de água equivalente a 2,5 vezes o peso do substrato, conforme Brasil (2009). Por fim, as sementes foram postas a germinar em câmara germinadora do tipo B.O.D., nas condições $30^{\circ} \mathrm{C}$ e luz constante.

As contagens foram feitas diariamente na primeira semana, até observação das primeiras sementes germinadas, a partir da segunda semana passaram a ser semanais, com avaliação das seguintes características: plântulas normais, anormais, sementes duras ou não germinadas e sementes mortas. Considerou-se semente germinada aquela cuja radícula fosse igual ou maior que $2 \mathrm{~mm}$. A germinação foi expressa em porcentagem e, adicionalmente, foi calculado o Índice de Velocidade de Germinação (IVG) segundo fórmula proposta por Maguire (1982).

O experimento teve duração de 150 dias após a semeadura, quando todos os tratamentos apresentavam o percentual de germinação estabilizado. Os dados das variáveis avaliadas foram submetidos à análise de variância (ANOVA) e as médias originadas dos tratamentos comparadas pelo teste de Scott Knott a 5\% de probabilidade. Houve necessidade de transformação em arc sen $(\sqrt{x+0,5}) / 100$ dos valores da variável mortalidade por não apresentarem distribuição normal e homogeneidade de variância dos resíduos, pressuposições essas necessárias para a realização da ANOVA. As análises foram realizadas com o auxílio dos softwares SAEG 9.1 (SAEG, 2007) e SISVAR (Ferreira, 2011).

\section{RESULTADOS E DISCUSSÃO}

O peso de mil sementes da Albizia pedicellaris foi de 30,76 g, ou seja, $1 \mathrm{~kg}$ possui 32.510 sementes, valor semelhante ao encontrado por Fowler et al. (2006) para Albizia hassleri, que foi de 36.656 sementes. O teor de umidade do lote utilizado no experimento foi de $8,33 \%$.

A análise de variância foi altamente significativa para germinação $(\mathrm{F}=79,15 ; \mathrm{p}<0,00001), \operatorname{IVG}(\mathrm{F}=110,79$; $\mathrm{p}<0,00001)$; plântulas normais $(\mathrm{F}=24,662 ; \mathrm{p}<0,00001)$; plântulas anormais $(\mathrm{F}=2,778 ; \mathrm{p}<0,0171)$; mortalidade $(\mathrm{F}=9,416 ; \mathrm{p}<0,00001)$, permitindo a diferenciação entre os tratamentos.

Os maiores percentuais germinativos foram encontrados nos tratamentos de escarificação mecânica seguida ou não da embebição em água por 6 e 12 horas, e imersão em ácido sulfúrico por 30 minutos (Tabela 1). Para esses tratamentos, a germinação variou de $85 \%$ a $95 \%$, sem diferença estatística significativa. A testemunha apresentou percentual germinativo de apenas 3\%, igualando-se estatisticamente ao choque térmico (4\%) e ao ácido sulfúrico 5 minutos (11\%). A imersão em ácido sulfúrico por 10 e 15 minutos promoveu a germinação de 15\% e 26\%, respectivamente, sem diferença estatística. A escarificação mecânica seguida de embebição por 24 horas promoveu $67 \%$ de germinação.

As sementes começaram a germinar aos quatro dias em todos os tratamentos, com exceção da testemunha (T1), que só germinou aos 88 dias (Figura 1). Os tratamentos: escarificação mecânica seguida ou não (T6) de embebição por 6 horas (T3) e por 12 horas (T4) alcançaram mais de $80 \%$ de germinação na primeira semana após a germinação. Já a imersão em ácido sulfúrico por 30 minutos (T10) alcançou mais de $80 \%$ somente na segunda semana, embora seus valores de velocidade de germinação não 
Tabela 1. Valores de porcentagem de germinação (G\%), Índice de Velocidade de Germinação (IVG), porcentagem de plântulas normais (PLN\%), de plântulas anormais (PLA\%) e de mortalidade (M\%) de sementes de Albizia pedicellaris submetidas a diferentes tratamentos pré-germinativos, após 150 dias de implantação do teste, em condições controladas.

Table 1. Values of germination percentage (G\%), Germination Speed Index (GSI), percentage of normal seedlings (PLN\%), abnormal seedlings (PLA\%) and mortality (M\%) of Albizia pedicellaris' seeds under different pregermination treatments after 150 days of deployment testing under controlled conditions.

\begin{tabular}{|clrrrrr}
\hline & & G\% & IVG & PLN\% & PLA \% & M\% \\
\hline T1 & Controle & $3,0^{\mathrm{d}}$ & $0,05^{\mathrm{c}}$ & $3,0^{\mathrm{c}}$ & $0^{\mathrm{b}}$ & $6,0^{\mathrm{b}}$ \\
\hline T2 & Choque térmico & $4,0^{\mathrm{d}}$ & $0,24^{\mathrm{c}}$ & $2,0^{\mathrm{c}}$ & $2,0^{\mathrm{b}}$ & $36,0^{\mathrm{a}}$ \\
\hline T3 & Escarificação mecânica e embebição por 6 horas & $95,0^{\mathrm{a}}$ & $15,97^{\mathrm{a}}$ & $84,0^{\mathrm{a}}$ & $11,0^{\mathrm{a}}$ & $5,0^{\mathrm{c}}$ \\
\hline T4 & Escarificação mecânica e embebição por 12 horas & $85,0^{\mathrm{a}}$ & $14,27^{\mathrm{a}}$ & $72,0^{\mathrm{a}}$ & $12,0^{\mathrm{a}}$ & $15,0^{\mathrm{b}}$ \\
\hline T5 & Escarificação mecânica e embebição por 24 horas & $67,0^{\mathrm{b}}$ & $11,05^{\mathrm{b}}$ & $48,0^{\mathrm{b}}$ & $19,0^{\mathrm{a}}$ & $33,0^{\mathrm{a}}$ \\
\hline T6 & Escarificação mecânica sem embebição & $88,0^{\mathrm{a}}$ & $14,74^{\mathrm{a}}$ & $83,0^{\mathrm{a}}$ & $5,0^{\mathrm{b}}$ & $12,0^{\mathrm{b}}$ \\
\hline T7 & Ácido sulfúrico - imersão por 5 minutos & $11,0^{\mathrm{d}}$ & $0,45^{\mathrm{c}}$ & $10,0^{\mathrm{c}}$ & $1,0^{\mathrm{b}}$ & $2,0^{\mathrm{c}}$ \\
\hline T8 & Ácido sulfúrico - imersão por 10 minutos & $15,0^{\mathrm{c}}$ & $0,60^{\mathrm{c}}$ & $9,0^{\mathrm{c}}$ & $6,0^{\mathrm{b}}$ & $1,0^{\mathrm{c}}$ \\
\hline T9 & Ácido sulfúrico - imersão por 15 minutos & $26,0^{\mathrm{c}}$ & $1,75^{\mathrm{c}}$ & $24,0^{\mathrm{c}}$ & $2,0^{\mathrm{b}}$ & $4,0^{\mathrm{c}}$ \\
\hline T10 & Ácido sulfúrico - imersão por 30 minutos & $93,0^{\mathrm{a}}$ & $13,21^{\mathrm{a}}$ & $86,0^{\mathrm{a}}$ & $7,0^{\mathrm{b}}$ & $7,0^{\mathrm{b}}$ \\
\hline
\end{tabular}

Médias seguidas pela mesma letra em cada característica não diferem entre si pelo Teste Scott-Knott a 5\% de probabilidade.

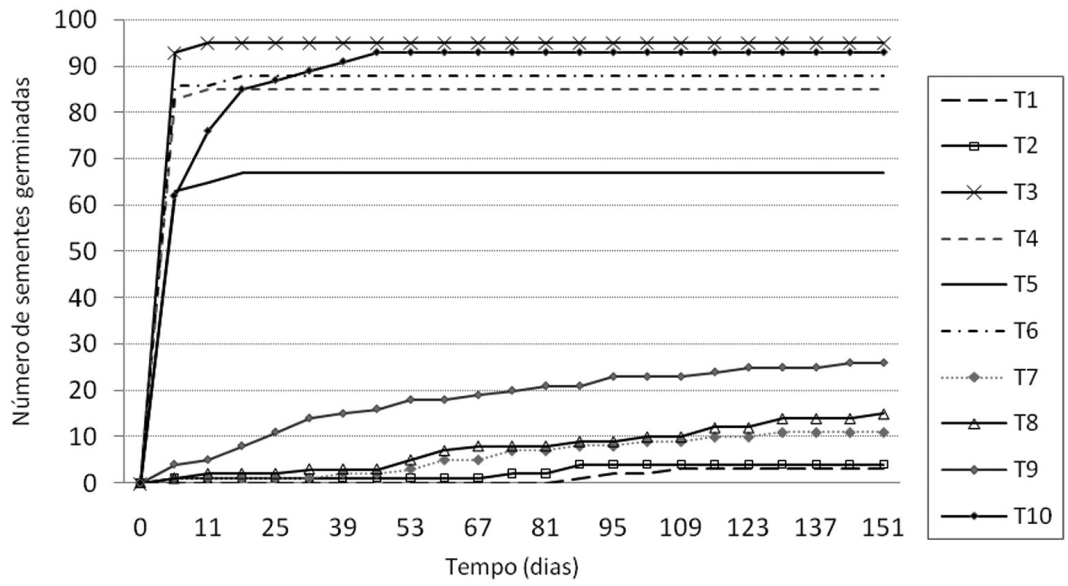

Figura 1. Tempo de germinação de sementes de Albizia pedicellaris submetidas a diferentes tratamentos pré-germinativos em condições controladas.

Figure 1. Time germination of Albizia pedicellaris' seeds subjected to different pre-germination treatments under controlled conditions.

tenham se diferenciado estatisticamente de T3, T4 e

T6. Esses tratamentos apresentaram os maiores índices de velocidade de germinação, com valores variando de 13,21 a 15,97 (Tabela 1 ).

O tratamento escarificação mecânica com embebição por 24 horas (T5) também alcançou rápida germinação logo na primeira semana, mas como teve baixa germinação $(67 \%)$ em relação aos melhores tratamentos, sua velocidade de germinação foi diferenciada estatisticamente do primeiro grupo.
Os tratamentos controle (T1), choque térmico (T2), e ácido sulfúrico 5 min (T7), 10 min (T8) e 15 min (T9) apresentaram aumento gradual da germinação com o tempo: após 80 dias ainda tinham sementes germinando (Figura 1).

O percentual de sementes mortas foi mais alto nos tratamentos com escarificação mecânica seguidos de embebição por 24 horas e choque térmico, chegando a $33 \%$ e $36 \%$ de mortalidade, respectivamente. No tratamento choque térmico, a exposição das sementes ao calor pode ter causado a morte do embrião, através 
da desnaturação de proteínas nas células e danos causados pela alta temperatura. Resultado semelhante foi encontrado para A. lebbeck (Sequeira et al., 2002).

A temperatura da água pode ser determinante na promoção da germinação. Para Albizia richardiana, espécie nativa da Ásia, o tratamento utilizando água quente com temperatura a $80^{\circ} \mathrm{C}$ por 10 minutos não danificou as sementes e promoveu a germinação de 96\%, em relação aos 49\% do controle (Azad et al., 2010). Para a espécie $A$. procera, a água quente a $80^{\circ} \mathrm{C}$ por 10 minutos elevou em aproximadamente $20 \%$ a germinação em relação à testemunha (Sajeevukumar et al., 1995; Azad et al., 2012). Para Albizia schimperiana, recomenda-se a imersão em água quente a $75{ }^{\circ} \mathrm{C}$ (Msanga \& Maghembe, 1986). Temperaturas de água de $60^{\circ} \mathrm{C}$ a $80^{\circ} \mathrm{C}$ se mostraram eficientes para superação da dormência da semente de A. falcataria, elevando a germinação de $10 \%$ para mais de $90 \%$, entretanto a elevação da temperatura para $100^{\circ} \mathrm{C}$ causou danos às sementes, reduzindo para $20 \%$ o percentual germinativo (Sajeevukumar et al., 1995). Seria interessante, com base nesses estudos, testar a quebra de dormência de A. pedicellaris a temperaturas mais baixas que a praticada neste trabalho $\left(80^{\circ} \mathrm{C}\right)$.

A embebição por 24 horas após escarificação das sementes também se mostrou danosa para as sementes. A entrada de água na semente ocorre em função da diferença do potencial hídrico entre o interior da semente e o meio. Se ocorrer em grande quantidade e rapidez, a entrada de água pode limitar o suprimento adequado de oxigênio para a semente durante o período de embebição, induzindo a alteração da via respiratória aeróbia para a fermentativa ou anaeróbia (Neumann et al., 1999). Como resultado, pode haver um decréscimo do vigor e mortalidade, conforme observado neste estudo. A alta mortalidade também pode ser atribuída ao ataque de microrganismos, cuja entrada pode ter sido facilitada pela escarificação. Semelhante resultado foi observado para sementes escarificadas de buriti (Mauritia flexuosa L.f.), seguida ou não de embebição em água (Seleguini et al., 2012). Entretanto, para outras espécies, como Moringa oleifera L. (Alves et al., 2005b) e Rollinia mucosa (Jacq.) Baill., (Ferreira et al., 2009), a germinação foi beneficiada pela embebição da semente em água por 24 horas.

A escarificação seguida de embebição por 6 horas (T3) apresentou apenas 5\% de sementes mortas, valor estatisticamente inferior à escarificação sem embebição (12\%) e à escarificação seguida de embebição por 12 horas (15\%).

A imersão em ácido sulfúrico por 30 minutos se mostrou eficiente para quebra de dormência de sementes de A. pedicellaris. Esse tempo é relativamente longo em relação ao utilizado de forma eficiente para outras espécies desse gênero, o que provavelmente está associado ao tegumento mais duro de A. pedicellaris. Albizia hassleri superou a dormência com a imersão das sementes em ácido sulfúrico por apenas 1 minuto (Fowler et al., 2006) e A. lebbeck, com a imersão por 1 a 5 minutos (Sequeira et al., 2002). A. falcataria e $A$. procera precisaram de 10 minutos de imersão (Sajeevukumar et al., 1995) e A. richardiana apresentou bons resultados com imersão em ácido sulfúrico por 20 minutos (Azad et al., 2010).

Para muitas espécies de outros gêneros e famílias já foi constatado o benefício na germinação com a imersão em ácido sulfúrico por períodos maiores que 20 minutos, denotando maior resistência dos tecidos germinativos a esse tratamento. Podem ser citados como exemplos as espécies Piptadenia viridiflora (Kunth) Benth, que se beneficiou com a imersão por 35 minutos em ácido sulfúrico (Santos et al., 2014), Lithraea molleoides (Vell.) Engl. demandou 20 minutos de imersão (Piveta et al., 2014) e Acacia mangium Willd, 90 minutos (Rodrigues et al., 2008).

Em viveiros, a utilização de ácido sulfúrico deve ser evitada. Nesses locais, a imersão em água quente e a escarificação mecânica são métodos preferenciais para superar a dormência tegumentar, por oferecerem menor risco de queimaduras para os trabalhadores que o ácido sulfúrico (Martins \& Nakagawa, 2008).

\section{CONCLUSÕES}

Os melhores tratamentos foram escarificação mecânica seguida ou não da embebição em água por 6 e por 12 horas e imersão em ácido sulfúrico por 30 minutos, pois promoveram os melhores valores de germinação e IVG, ao mesmo tempo que tiveram um baixo percentual de mortalidade.

\section{STATUS DA SUBMISSÃO}

Recebido: 12 jul., 2014

Aceito: 8 nov., 2015 
AUTOR(ES) PARA CORRESPONDÊNCIA

\section{Juliana Müller Freire}

Laboratório de Leguminosas, Embrapa Agrobiologia, BR 465, Km 07, CEP 23890-000, Seropédica, RJ, Brasil

e-mail: juliana.muller@embrapa.br

\section{REFERENNCIAS}

Alves EU, Bruno RDLA, Oliveira AD, Alves AU, Paula $\mathrm{RD}$. Influência do tamanho e da procedência de sementes de Mimosa caesalpiniifolia Benth. sobre a germinação e vigor. Revista Árvore 2005a; 29(6): 877-885. http://dx.doi. org/10.1590/S0100-67622005000600006.

Alves MDCS, Medeiros S Fo, Bezerra AME, Oliveira VCD. Germinação de sementes e desenvolvimento de plântulas de Moringa oleifera L. em diferentes locais de germinação e submetidas à pré-embebição. Ciência e Agrotecnologia 2005b; 29(5): 1083-1087. http://dx.doi. org/10.1590/S1413-70542005000500025.

Azad S, Biswas RK, Matin A. Seed germination of Albizia procera (Roxb.) Benth. in Bangladesh: a basis for seed source variation and pre-sowing treatment effect. Forestry Studies in China 2012; 14(2): 124-130. http://dx.doi. org/10.1007/s11632-012-0209-z.

Azad S, Paul NK, Matin A. Do pre-sowing treatments affect seed germination in Albizia richardiana and Lagerstroemia speciosa? Frontiers of Agriculture in China 2010; 4(2): 181-184. http://dx.doi.org/10.1007/s11703-010-0100-4.

Berger APDA, Ranal MA, Santana DGD. Variabilidade na dormência relativa dos diásporos de Lithraea molleoides (Vell.) Eng. Ciência Florestal 2014; 24(2): 325-337. http:// dx.doi.org/10.5902/1980509814570.

Botezelli L, Davide AC, Malavasi MM. Características dos frutos e sementes de quatro procedências de Dipteryx alata Vogel (Baru). Cerne 2000; 6(1): 9-18.

Brasil. Ministério da Agricultura e Reforma Agrária. Regras para análise de sementes. Brasília: Ministério da Agricultura; 2009.

Costa PA, Lima ALS, Zanella F, Freita H. Quebra de dormência em sementes de Adenanthera pavonina L. Pesquisa Agropecuária Tropical 2010; 40(1): 83-88.

Cruz ED, Carvalho JEU, Queiroz RJB. Scarification with sulphuric acid of Schizolobium amazonicum Huber ex Ducke seeds - Fabaceae. Scientia Agricola 2007; 64(3): 308-313. http://dx.doi.org/10.1590/S0103-90162007000300017.

Faria SM. Occurrence and rhizobial selection for legume trees adapted to acid soils. In: Evans DO, Szott LT, editores. Nitrogen-fixing trees for acid soils. Morrilton: Arkansas; 1995.
Ferreira DF. Sisvar: a computer statistical analysis system. Ciência e Agrotecnologia 2011; 35(6): 1039-1042.

Ferreira M, Santos MRA, Gonçalves E, Alves EU, Bruno RLA. Superação de dormência em sementes de biribá (Rollinia mucosa (Jacq.) Baill). Revista Brasileira de Sementes 2009; 31(4): 95-99. http://dx.doi.org/10.1590/ S0101-31222009000400011.

Floriano EP. Armazenamento de sementes florestais. Santa Rosa: ANORGS, 2004. Caderno didático.

Fowler JAP, Carpanezzi AA, Zuffellato-Ribas KC. Tecnologia para o manejo adequado de sementes de farinha-seca. Boletim de Pesquisa Florestal 2006; 53: 195-208.

Franco AA, Dias LE, Faria SM, Campello EFC, Silva EMR. Uso de leguminosas florestais noduladas e micorrizadas como agentes de recuperação e manutenção da vida no solo: um modelo tecnológico. Oecologia Brasiliensis 1995; 1(01):459-467. http://dx.doi.org/10.4257/oeco.1995.0101.23.

Funk VA, Berry PE, Alexander S, Hollowell TH, Kelloff CL. Checklist of the plants of the Guiana Shield (Venezuela: Amazonas, Bolivar, Delta Amacuro; Guyana, Surinam, French Guiana). Contributions from the United States National Herbarium 2007; 55: 1-584.

Giachini RM, Lobo F, Albuquerque MCF, Ortíz CER. Influência da escarificação e da temperatura sobre a germinação de sementes de Samanea tubulosa (Benth.) Barneby \& J.W. Grimes (sete cascas). Acta Amazonica 2010; 40(1): 75-80. http://dx.doi.org/10.1590/S004459672010000100010 .

Hokche O, Berry PE, Huber O. Nuevo Catálogo de la Flora Vascular de Venezuela. Caracas: Fundación Instituto Botánico de Venezuela; 2008.

Iganci JRV. Albizia in Lista de Espécies da Flora do Brasil [online]. Rio de Janeiro: Jardim Botânico do Rio de Janeiro; 2014. [citedo 2014 Abr 10]. Available from: http:// floradobrasil.jbrj.gov.br/jabot/floradobrasil/FB82618

Jorgensen PM, León-Yánez S. Catalogue of the vascular plants of Ecuador. St. Louis: Missouri Botanical Garden Press; 1999.

Kissmann C, Scalon SPQ, Mussury RM, Robaina AD. Germinação e armazenamento de sementes de Albizia hasslerii (Chod.) Burkart. Revista Brasileira de Sementes 2009; 31(2): 104-115. http://dx.doi.org/10.1590/S010131222009000200012 .

Ladeia ES, Coelho MFB, Azevedo RAB, Albuquerque MCF. Procedência do fruto e substratos na germinação de sementes de Pseudobombax longiflorum (Mart. et Zucc.) A. Robyns. Pesquisa Agropecuária Tropical 2012; 42(2): 174180. http://dx.doi.org/10.1590/S1983-40632012000200009.

Lorenzi H. Árvores brasileiras: manual de identificação e cultivo de plantas arbóreas nativas do Brasil. Nova Odessa: Editora Plantarum; 2002.

Maguire JD. Speed of germination-aid in selection and evaluation for seedling emergence and vigor. Crop Science 
1982; 2(2): 176-177. http://dx.doi.org/10.2135/cropsci19 62.0011183X000200020033x.

Martins CC, Nakagawa J. Germinação de sementes de Stryphnodendron adstringens (Mart.) Coville de diferentes origens submetidas a tratamentos para superação de dormência. Revista Árvore 2008; 32(6): 1059-1067. http:// dx.doi.org/10.1590/S0100-67622008000600011.

Mayer AM, Poljakoff-Mayber A. The germination of seeds. 3. ed. Oxford: Pergamon Press; 1982.

Mello JT. Coleta, propagação e desenvolvimento inicial de espécies de cerrado. In: Sano SM, Almeida SP, editors. Cerrado: ambiente e flora. Planaltina: Empresa Brasileira de Pesquisa Agropecuária; 1998.

Msanga HP, Maghembe JA. Effect of hot water and chemical treatments on the germination of Albizia schimperana seed. Forest Ecology and Management 1986; 17(2-3): 137 146. http://dx.doi.org/10.1016/0378-1127(86)90106-4.

Neumann G, Preißler M, Azaizeh HA, Romheld V. Thiamine (vitamin B1) deficiency in germinating seeds of Phaseolus vulgaris L. exposed to soaking injury. Journal of Plant Nutrition and Soil Science 1999; 162(3): 295-300. http://dx.doi. org/10.1002/(SICI)1522-2624(199906)162:3<295::AIDJPLN295>3.0.CO;2-R.

Piveta G, Muniz MDFB, Reiniger LRS, Dutra CB, Pacheco C. Qualidade sanitária e fisiológica de sementes de AroeiraPreta (Lithraea molleoides) submetidas a métodos de superação de dormência. Revista Brasileira de Sementes 2014; 31(2): 104-115.

Rodrigues APDAC, Kohl MC, Pedrinho DR, Arias, ERA, Favero S. Tratamentos para superar a dormência de sementes de Tratamentos para superar a dormência de sementes de Acacia mangium Willd. Acta Scientiarum Agronomy 2008; 30(2): 279-283.

Rolston MP. Water impermeable seed dormancy. Botanical Review 1978; 44(3): 365-396. http://dx.doi.org/10.1007/ BF02957854.

Sajeevukumar B, Sudhakara K, Ashokan PK, Gopikumar K. Seed dormancy and germination in Albizia falcataria and Albizia procera. Journal of Tropical Forest Science 1995; 7(3): 371-382.

Santos ALF, Freire JM, Pina-Rodrigues FCM. Avaliação de métodos para superação de dormência de sementes de leguminosas arbóreas utilizadas na recuperação de áreas degradadas. Serepédica: Embrapa Agrobiologia; 2011. Boletim de Pesquisa e Desemvolvimento n. 76.

Santos JL, Matsumoto SN, D'Arêde LO, Viana AES. Superação da dormência tegumentar de sementes de Piptadenia viridiflora (Kunth) Benth pela escarificação química. Bioscience Journal 2014; 30(6): 1642-1651.

Seleguini A, Camilo YMV, Souza ERB, Martins ML, Belo APM, Fernandes AL. Superação de dormência em sementes de buriti por meio da escarificação mecânica e embebição. Revista Agro@mbiente On-line 2012; 6(3): 235-241.

Sequeira F, Silva AJC, Carpanezzi AA, Fowler JAP. Quebra de dormência de sementes de Albizia polycephala (Benth.) Killip. In: I Evento de Iniciação Científica da Embrapa Florestas; 2002; Colombo. Colombo: Embrapa Florestas; 2002. p. 14.

Sistema para Análises Estatísticas - SAEG. SAEG Versão 9.1: Fundação Arthur Berrnardes. Viçosa: UFV; 2007.

Souza ERB, Zago R, Garcia J, Farias JG, Carvalho SEM, Barroso MR. Efeito de métodos de escarificação do tegumento em sementes de Leucaena diversifolia L. Pesquisa Agropecuária Tropical 2007; 37(3): 142-146.

Souza TV, Torres IC, Steiner N, Paulilo MTS. Seed dormancy in tree species of the Tropical Brazilian Atlantic Forest and its relationships with seed traits and environmental conditions. Brazilian Journal of Botany 2015; 38(2): 243-264. http://dx.doi.org/10.1007/s40415-014-0129-3.

Steyermark J. Flora of the Venezuelan Guayana Project [online]. 1995. [cited $2014 \mathrm{Fev} 12$ ]. Availabe from: http:// www.tropicos.org/Reference/158

Villiers TA. Seed dormancy. In: Kozlowsky TT, editor. Seed biology. New York: Academic Press; 1972. 